\title{
Hall MHD and Electron Inertia Effects in Current Sheet Formation at a Magnetic Neutral Line
}

\author{
Yuri E. Litvinenko and Liam C. McMahon* \\ Department of Mathematics, University of Waikato, Private Bag 3105, Hamilton, \\ New Zealand. \\ Received 16 September 2014; Accepted (in revised version) 6 March 2015.
}

\begin{abstract}
An exact self-similar solution is used to investigate current sheet formation at a magnetic neutral line in incompressible Hall magnetohydrodynamics. The collapse to a current sheet is modelled as a finite-time singularity in the solution for electric current density at the neutral line. We establish that a finite-time collapse to the current sheet can occur in Hall magnetohydrodynamics, and we find a criterion for the finite-time singularity in terms of the initial conditions. We derive an asymptotic solution for the singularity formation and a formula for the singularity formation time. The analytical results are illustrated by numerical solutions, and we also investigate an alternative similarity reduction. Finally, we generalise our solution to incorporate resistive, viscous and electron inertia terms.
\end{abstract}

AMS subject classifications: 76W05, 85A30

Key words: Finite-time singularities, Hall MHD, magnetic reconnection, current sheet formation.

\section{Introduction}

The Hall effect can significantly modify plasma behaviour $[18,42]$. In particular, the magnetic reconnection rates predicted by resistive magnetohydrodynamic models $[27,38]$ are too slow to explain reconnection in laboratory and astrophysical plasmas $[1,44,46]$. Numerical simulations demonstrate that including the Hall terms can speed up reconnection $[2,3,12,29]$. Moreover, numerical results are consistent with analytical models that quantify the role of the Hall effect in steady reconnection [24,33, 41].

How quickly does a current sheet form in a weakly collisional plasma, and what is the role of the Hall effect in the process? Singularity formation models, which identify the sheet formation with the growth of the electric current density, make it possible to describe the current sheet formation using exact analytical solutions. Exact self-similar solutions in both ideal and resistive magnetohydrodynamics (MHD) have been found to exhibit both exponential growth of the current density [7,39] and finite-time collapse to a singularity [30]. The main limitation of these open-geometry solutions is that they do not

\footnotetext{
${ }^{*}$ Corresponding author. Email address: 1cm21@students.waikato.ac.nz (L. C. McMahon) 
predict the thickness of the emerging current sheet. However, the predicted exponential behaviour was confirmed by numerical simulations [16,37], and analytical arguments show that these solutions should evolve exponentially unless a singularity is driven by an imposed pressure [21].

Here we investigate a self-similar solution for current sheet formation in Hall MHD, i.e. when the Hall effect is included. The fundamental equations are presented in Section 2. In Sections 3 and 4, we generalise previous studies [23] by considering a general set of initial conditions and derive a criterion for the formation of a finite-time singularity. The new solution reduces to the exponentially evolving MHD solution upon setting the Hall term to zero. In Section 5, we discuss an alternative approach [32] to the singularity formation in Hall MHD. In Section 6, we generalise our new solution to incorporate resistive, viscous and electron inertia effects. We discuss the results in Section 7.

\section{Generalised Ohm's Law and MHD Equations}

The incompressible MHD equations in dimensionless form are given by a generalised Ohm's law [28]

$$
\mathbf{E}+\mathbf{v} \times \mathbf{B}=\eta \mathbf{J}+d_{i}\left(\mathbf{J} \times \mathbf{B}-\nabla p_{e}\right)+d_{e}^{2}\left[\partial_{t} \mathbf{J}+(\mathbf{v} \cdot \nabla) \mathbf{J}+(\mathbf{J} \cdot \nabla) \mathbf{v}\right],
$$

the equation of motion

$$
\partial_{t} \mathbf{v}+(\mathbf{v} \cdot \nabla) \mathbf{v}=-\nabla p+\mathbf{J} \times \mathbf{B}+v \nabla^{2} \mathbf{v}
$$

the continuity equation

$$
\nabla \cdot \mathbf{v}=0
$$

and electromagnetic equations

$$
\begin{aligned}
& \nabla \cdot \mathbf{B}=0, \\
& \mathbf{J}=\nabla \times \mathbf{B}, \\
& \nabla \times \mathbf{E}=-\partial_{t} \mathbf{B},
\end{aligned}
$$

where $\partial_{t}$ denotes partial differentiation with respect to the time $t, \mathbf{v}$ is the plasma velocity, $\mathbf{B}$ the magnetic field, $\mathbf{J}$ the electric current density, $\mathbf{E}$ the electric field, and the total plasma pressure $p$ and electron pressure $p_{e}$ are scalar fields [43]. Here we use Gaussian cgs units for consistency with other theoretical studies. The length and magnetic field are scaled by typical reference values $L$ and $B_{0}$, the velocity $\mathbf{v}$ is normalised by the Alfvén speed $v_{A}=B_{0} / \sqrt{4 \pi \rho}$ where $\rho \simeq m_{i} n$ is the mass density (with the relation $m_{e} \ll m_{i}$ between the electron and ion masses and $n$ their common particle number density), the time is normalised by the Alfvén time $t_{A}=L / v_{A}$, and the assumed constant resistivity $\eta$ and viscosity $v$ by $4 \pi L v_{A} / c^{2}$ and $L v_{A}$, respectively (where $c$ is the speed of light). The adoption of the scalar viscosity term in the equation of motion, as opposed to a more general anisotropic viscous stress tensor, is justified in the vicinity of a magnetic null where the magnetic field 
is weak (e.g. see Ref. [19] and references therein). The collisionless effects are quantified by the ion skin depth $d_{i}=c /\left(L \omega_{p i}\right)$ and the electron skin depth $d_{e}=c /\left(L \omega_{p e}\right)$, involving the ion and electron plasma frequencies $\omega_{p i}=\left(4 \pi n e^{2} / m_{i}\right)^{1 / 2}$ and $\omega_{p e}=\left(4 \pi n e^{2} / m_{e}\right)^{1 / 2}$ where $e$ denotes the proton charge.

The Hall effect is significant in Eq. (2.1) if $d_{i}|\mathbf{J} \times \mathbf{B}| \gtrsim|\eta \mathbf{J}|$. In order to estimate the value of $d_{i}$ at which the Hall term becomes significant during reconnection, note that there is a large gradient in the planar magnetic field $B_{p l}$ but the out-of-plane $z$-component of the magnetic field changes relatively slowly [44]. From Eq. (2.5), we estimate that $J_{p l} \sim 1$ and so $\left|\mathbf{J}_{p l} \times \mathbf{B}_{p l}\right| \simeq B_{p l}$ whereas $J_{z} \sim B_{p l} / l$ (where $l$ denotes the current sheet thickness), so that $E_{z} \sim \eta B_{p l} / l \sim d_{i} B_{p l}$. A typical Sweet-Parker length scale is $l \sim \eta^{1 / 2}$, which implies that the Hall effect becomes significant in reconnection when $d_{i}^{2} \gtrsim \eta[9]$. Physically, $d_{i}$ gives the dimensionless thickness of a current sheet determined by collisionless effects [17].

In ideal Hall MHD, we set $d_{e}=v=\eta=0$ in the generalised Ohm's law (2.1) and the equation of motion (2.2). We assume a " $2.5 \mathrm{D}$ model", in which all quantities are considered in three dimensions but there is no dependence on the $z$-coordinate $\left(\partial_{z}=0\right)$. The incompressibility equation (2.3) then dictates that

$$
\mathbf{v}(x, y, t)=\nabla \phi \times \hat{\mathbf{z}}+W \hat{\mathbf{z}},
$$

where the velocity potential $\phi$ corresponds to the planar flow and $W$ is the out-of-plane velocity component ( $\hat{\mathbf{z}}$ is the unit vector in the $z$-direction). Similarly, to satisfy Eq. (2.4) we introduce the flux function $\psi$ to represent the planar magnetic field.

$$
\mathbf{B}(x, y, t)=\nabla \psi \times \hat{\mathbf{z}}+Z \hat{\mathbf{z}},
$$

where $Z$ is the axial magnetic field. The pressure terms do not contribute to the $z$-components of Eqs. (2.1) and (2.2), and to eliminate the pressure terms in the $x$ and $y$ components we take the curl of those equations. Eqs. (2.1)-(2.6) thus simplify to the following system [10]:

$$
\begin{aligned}
& \partial_{t} \psi+[\psi, \phi]=d_{i}[\psi, Z], \\
& \partial_{t} Z+[Z, \phi]=[W, \psi]+d_{i}\left[\nabla^{2} \psi, \psi\right], \\
& \partial_{t} W+[W, \phi]=[Z, \psi], \\
& \partial_{t}\left(\nabla^{2} \phi\right)+\left[\nabla^{2} \phi, \phi\right]=\left[\nabla^{2} \psi, \psi\right],
\end{aligned}
$$

where the Poisson bracket notation is typified by

$$
[\psi, \phi]=\partial_{x} \psi \partial_{y} \phi-\partial_{y} \psi \partial_{x} \phi .
$$

\section{Self-Similar Solutions}

Dungey $[13,14]$ was the first to consider the collapse of a magnetic X-point to a current sheet. Chapman \& Kendall [7, 8] then obtained an exact solution for two-dimensional X-type collapse in an incompressible infinitely conducting plasma (see also Ref. [37]). 
A key feature of their solution is an exponential growth of the X-point magnetic field. Uberoi $[39,40]$ noted the validity of the solution for finite conductivity, whereas Ref. [20] obtained a solution for a compressible plasma. Magnetic collapse has also been studied in three dimensions [4] (see Ref. [25] for a review of related topics). In incompressible MHD, a two-dimensional solution predicted a finite-time collapse to the current sheet [30]. However, numerical simulations have shown an exponential flattening of the X-point in ideal incompressible MHD [16,37]. Ref. [21] proved that in general a finite-time collapse to a current sheet cannot occur in planar incompressible MHD flows unless a singularity is pressure-driven (e.g. Ref. [30]).

We solve the ideal Hall MHD equations via similarity reduction, to obtain self-similar solutions that generalise those of purely resistive MHD. The nonlinear Hall term in the curl of Eq. (2.1) $d_{i} \nabla \times(\mathbf{J} \times \mathbf{B})$ vanishes in classical resistive MHD. On dimensional grounds, we may expect that a strong Hall effect should lead to the plasma evolution on a time scale of order $d_{i}^{-1}$. The detailed solution in the next section confirms this scaling and leads to an expression for the current sheet formation time in Hall MHD. For some initial conditions, it turns out that the solution contains a finite time singularity. The implied unlimited growth of the energy density in the vicinity of the magnetic null is formally possible in open-geometry solutions because of energy flux from the outside. Although such singular self-similar solutions only hold locally as low-order Taylor expansions and break down after a finite time, the predicted singularity formation time can be useful in quantifying the role of the Hall effect and the initial conditions in the current sheet formation.

As in incompressible MHD in two dimensions, we reduce the system (2.9)-(2.12) to a system of ordinary differential equations yielding solutions describing a hyperbolic planar magnetic field driven by a stagnation-point flow:

$$
\begin{aligned}
& \psi=\alpha(t) x^{2}-\beta(t) y^{2} \\
& \phi=-\gamma(t) x y
\end{aligned}
$$

For the axial velocity $W$ and magnetic field $Z$, we assume

$$
\begin{aligned}
& W=f(t) x^{2}+g(t) y^{2}, \\
& Z=h(t) x y
\end{aligned}
$$

where the form of the axial magnetic field corresponds to the well-known quadrupolar structure in Hall magnetic reconnection [34,35, 43]. On substituting Eqs. (3.1)-(3.4) into Eqs. (2.9)-(2.12) we get

$$
\begin{aligned}
& \dot{\alpha}-2 \alpha\left(\gamma+d_{i} h\right)=0, \\
& \dot{\beta}+2 \beta\left(\gamma+d_{i} h\right)=0, \\
& \dot{f}-2 \gamma f+2 \alpha h=0, \\
& \dot{g}+2 \gamma g+2 \beta h=0, \\
& \dot{h}+4 \alpha g+4 \beta f=0,
\end{aligned}
$$


where the overdot represents differentiation with respect to the dimensionless time.

Substitution into the equation of motion (2.2) and integration then produces the inviscid $(v=0)$ plasma pressure profile

$$
p(x, y, t)=-\frac{1}{2} h^{2} x^{2} y^{2}+\frac{1}{2}\left[\gamma^{2}-\dot{\gamma}-4 \alpha(\alpha-\beta)\right] x^{2}+\frac{1}{2}\left[-\gamma^{2}-\dot{\gamma}+4 \beta(\alpha-\beta)\right] y^{2} .
$$

Setting $h(t)=0$ leads to the MHD result without the Hall effect.

\section{Collapse to a Current Sheet in Hall MHD}

For a general set of initial conditions

$$
\alpha(0)=\alpha_{0}, \beta(0)=\beta_{0}, \gamma(0)=\gamma_{0}, f(0)=f_{0}, g(0)=g_{0}, h(0)=h_{0},
$$

integration of Eqs. (3.5)-(3.9) yields

$$
\begin{aligned}
& \alpha \beta=\alpha_{0} \beta_{0}, \\
& \alpha+d_{i} f=\left(\alpha_{0}+d_{i} f_{0}\right) \exp (2 \Gamma), \\
& \beta-d_{i} g=\left(\beta_{0}-d_{i} g_{0}\right) \exp (-2 \Gamma), \\
& h^{2}-4 f g=h_{0}^{2}-4 f_{0} g_{0},
\end{aligned}
$$

where $\Gamma=\int_{0}^{t} \gamma\left(t^{\prime}\right) \mathrm{d} t^{\prime}$. These equations generalise those derived in Ref. [23] for the case $\gamma=$ const. On differentiating Eq. (3.9), we obtain an equation for $h(t)$ :

$$
\ddot{h}+4(\dot{\alpha} g+\alpha \dot{g}+\dot{\beta} f+\beta \dot{f})=0,
$$

which from (3.5)-(3.9) simplifies to

$$
\ddot{h}+8 h\left[d_{i}(\alpha g-\beta f)-2 \alpha_{0} \beta_{0}\right]=0 .
$$

In order to express $(\alpha g-\beta f)$ in terms of $h$, we note that (4.2) and (4.3) yield

$$
\left(\alpha+d_{i} f\right)\left(\beta-d_{i} g\right)=\left(\alpha_{0}+d_{i} f_{0}\right)\left(\beta_{0}-d_{i} g_{0}\right) .
$$

So on expanding the left-hand side and using Eq. (4.1) we get

$$
d_{i}(\alpha g-\beta f)=\alpha_{0} \beta_{0}-d_{i}^{2} f g-\left(\alpha_{0}+d_{i} f_{0}\right)\left(\beta_{0}-d_{i} g_{0}\right) .
$$

Next we use Eq. (4.4) to eliminate $f g$, to obtain

$$
\ddot{h}-2 d_{i}^{2} h^{3}-a^{2} h=0,
$$

where $a^{2}$ is defined as

$$
a^{2}=-2\left[4 d_{i}\left(\alpha_{0} g_{0}-\beta_{0} f_{0}\right)-8 \alpha_{0} \beta_{0}+d_{i}^{2} h_{0}^{2}\right] .
$$






Figure 1: $U(h)$ vs. $h$.

Note that Eq. (4.6) is valid for any $\gamma(t)$.

A finite-time collapse to a current sheet occurs if a finite-time singularity is present in the solution, i.e. if $h(t) \rightarrow \infty$ as $t \rightarrow t_{s}$. A singularity criterion can be obtained using a mechanical analogy. Let us rewrite Eq. (4.6) as

$$
\ddot{h}+U^{\prime}(h)=0,
$$

where $U(h)$ is analogous to potential energy in mechanics (see Fig. 1). Thus we can view the solution to Eq. (4.6) as particle motion in this potential, and integration of Eq. (4.8) yields an analogue of energy conservation:

$$
\frac{1}{2} \dot{h}^{2}=-U(h),
$$

where

$$
U(h)=-\frac{1}{2}\left(d_{i}^{2} h^{4}+a^{2} h^{2}\right)+\frac{1}{2}\left(d_{i}^{2} h_{0}^{4}+a^{2} h_{0}^{2}\right)-8\left(\alpha_{0} g_{0}+\beta_{0} f_{0}\right)^{2}
$$

is a quartic function that tends to $-\infty$ for large $h$. On setting $U^{\prime}\left(h_{\max }\right)=0$, we find

$$
h_{\max }^{2}=-\frac{a^{2}}{2 d_{i}^{2}} .
$$

The solution $h(t)$ remains near the origin if the following three conditions are satisfied:

- $U(h)$ has a local minimum;

- $h(t)$ has upper and lower bounds $\pm h_{\max }$, i.e. $h(t)$ does not escape the local potential well; and

- at $t=0, h(t)=h_{0}$ lies between the upper and lower bounds $\pm h_{\max }$.

Near the origin $U(h) \simeq$ const $-a^{2} h^{2} / 2$. To satisfy the first condition, we must have $a^{2}<0$. To satisfy the second condition, we require that $\dot{h}^{2} \leq 0$ at the bounds - or equivalently 
that $U\left(h_{\max }\right) \geq 0$. After some algebra, Eq. (4.10), given $h_{\max }$ from Eq. (4.11) and $a^{2}$ from Eq. (4.7), yields

$$
\alpha_{0} \beta_{0}\left(\alpha_{0}+d_{i} f_{0}\right)\left(\beta_{0}-d_{i} g_{0}\right) \geq 0 .
$$

The third condition implies $h_{0}^{2} \leq h_{\text {max }}^{2}$, so that

$$
d_{i}\left(\alpha_{0} g_{0}-\beta_{0} f_{0}\right)-2 \alpha_{0} \beta_{0} \geq 0 \text {. }
$$

Inequality (4.13) is in fact a stronger condition than $a^{2}<0$, so only the last two conditions on the initial values of $\alpha, \beta, f$ and $g$ must be satisfied for the solution $h(t)$ to remain near the origin. Our self-similar solution will therefore not contain a finite-time singularity if the initial conditions $\alpha_{0}, \beta_{0}, f_{0}$ and $g_{0}$ are such that inequalities (4.12) and (4.13) are satisfied. Significantly, these conditions do not contain $\gamma(t)$ and $h_{0}$. If either of (4.12) or (4.13) is not satisfied, the solution develops a singularity. Thus in sharp contrast to the exponential collapse in the absence of the Hall effect, in Hall MHD the collapse to a current sheet can occur in a finite time. For example, (4.13) is not satisfied for the particular case considered in Ref. [23] where $\alpha_{0}=\beta_{0}, \gamma_{0}=0.5$ and $f_{0}=g_{0}=0$. We also note that this criterion predicts exponential evolution for the case $d_{i}=0$.

Eq. (4.6) can be solved in terms of Jacobi elliptic functions. However, these solutions are difficult to work with, so we approximate the collapse solution in terms of elementary functions assuming $a^{2}>0$. Near the singularity, we let each variable be dependent on a power of $\tau=\left(t_{s}-t\right)$ where $t_{s}$ is the singularity time, and then let $\tau \rightarrow 0$. Due to the hyperbolic shape of the flow, either $\alpha \rightarrow \infty$ and $\beta \rightarrow 0$ or $\alpha \rightarrow 0$ and $\beta \rightarrow \infty$. Now $\ddot{h} \simeq 2 d_{i}^{2} h^{3}$ for large $h$, so $h$ is proportional to $\pm \tau^{-1}$. On substituting $d_{i} h=\tau^{-1}$ into Eqs. (3.5), (3.6), (4.3) and (4.4) with $\tau \rightarrow 0$ and $\Gamma \rightarrow \Gamma_{s}$ (the value at the singularity) and balancing the leading-order terms, we have

$$
\begin{aligned}
& \alpha \simeq \frac{1}{4\left(\beta_{0}-d_{i} g_{0}\right)} \exp \left(2 \Gamma_{s}\right) \tau^{-2}, \\
& \beta \simeq 4 \alpha_{0} \beta_{0}\left(\beta_{0}-d_{i} g_{0}\right) \exp \left(-2 \Gamma_{s}\right) \tau^{2}, \\
& d_{i} f \simeq \frac{-1}{4\left(\beta_{0}-d_{i} g_{0}\right)} \exp \left(2 \Gamma_{s}\right) \tau^{-2}, \\
& d_{i} g \simeq-\left(\beta_{0}-d_{i} g_{0}\right) \exp \left(-2 \Gamma_{s}\right), \\
& d_{i} h \simeq \tau^{-1},
\end{aligned}
$$

or

$$
\begin{aligned}
& \alpha \simeq 4 \alpha_{0} \beta_{0}\left(\alpha_{0}+d_{i} f_{0}\right) \exp \left(2 \Gamma_{s}\right) \tau^{2}, \\
& \beta \simeq \frac{1}{4\left(\alpha_{0}+d_{i} f_{0}\right)} \exp \left(-2 \Gamma_{s}\right) \tau^{-2}, \\
& d_{i} f \simeq\left(\alpha_{0}+d_{i} f_{0}\right) \exp \left(2 \Gamma_{s}\right), \\
& d_{i} g \simeq \frac{1}{4\left(\alpha_{0}+d_{i} f_{0}\right)} \exp \left(-2 \Gamma_{s}\right) \tau^{-2}, \\
& d_{i} h \simeq-\tau^{-1} .
\end{aligned}
$$


This requires that $\Gamma(t) \rightarrow \Gamma_{s}=\int_{0}^{t_{s}} \gamma\left(t^{\prime}\right) \mathrm{d} t^{\prime}$, assuming that the integral converges. It is reasonable to assume that $\gamma(t)$ is non-singular because $\gamma(t)$ represents the driving flow.

Next, we use asymptotic analysis to determine the singularity time $t_{s}$. For small time we have that $\left(d_{i} h\right)^{2} \ll 1$, and Eq. (4.6) simplifies to $\ddot{h} \simeq a^{2} h$. The solution is thus

$$
h(t) \simeq h_{0} \cosh (a t)+\frac{\dot{h}_{0}}{a} \sinh (a t),
$$

where $h_{0}=h(0)$ and $\dot{h}_{0}=-4\left(\alpha_{0} g_{0}+\beta_{0} f_{0}\right)$. For large time $t$ we use Eqs. (4.9) and (4.10). Near the singularity we have $h \rightarrow \infty$, so a constant term can be neglected. Integrating the resulting equation

$$
\dot{h}^{2} \simeq d_{i}^{2} h^{4}+a^{2} h^{2}
$$

gives

$$
h(t) \simeq \frac{2 a^{2} k \exp (a t)}{1-\left(d_{i} a k\right)^{2} \exp (2 a t)},
$$

where the integration constant $k$ specifies the singularity time $t_{s}$ such that $h(t) \rightarrow \infty$ as $t \rightarrow t_{s}$.

An intermediate asymptotic solution follows from Eqs. (4.24) and (4.26) by requiring that they coincide in the range $a^{-1}<t<t_{s}$, which yields an equation for $k$ :

$$
\frac{2 a^{2} k}{1-\left(d_{i} a k\right)^{2}}=\frac{h_{0}}{2}+\frac{\dot{h}_{0}}{2 a} \text {. }
$$

On solving the resulting quadratic equation and assuming $\left(h_{0}+\dot{h}_{0} / a\right)\left(d_{i} a\right)^{2} \ll 1$, we obtain

$$
k=\frac{1}{4 a^{2}}\left(h_{0}+\frac{\dot{h}_{0}}{a}\right),
$$

which leads to the intermediate asymptotic solution valid for all time:

$$
h(t) \simeq\left(h_{0} \cosh (a t)+\frac{\dot{h}_{0}}{a} \sinh (a t)\right)\left[1-\frac{d_{i}^{2}}{16 a^{2}}\left(h_{0}+\frac{\dot{h}_{0}}{a}\right)^{2} \exp (2 a t)\right]^{-1} .
$$

The singularity time $t_{s}$ in terms of the initial values is therefore

$$
t_{s}=\frac{1}{2 a} \ln \left[\frac{16 a^{2}}{d_{i}^{2}}\left(h_{0}+\frac{\dot{h}_{0}}{a}\right)^{-2}\right] \text {. }
$$

When $a=4$ and $\dot{h}_{0}=0$ we recover the case considered in Ref. [23]. It is also notable that the scaling $t_{s} \sim a^{-1} \sim\left(d_{i} h_{0}\right)^{-1}$ is consistent with the dimensional estimate when the Hall effect is strong. Our formula for the singularity formation time quantifies the role of the Hall effect and initial conditions in the current sheet formation.

We illustrate the criteria (4.12) and (4.13) by plotting the numerical solutions of the system (3.5)-(3.9) with varied initial conditions (Figs. 2-4). There are six variables in our 

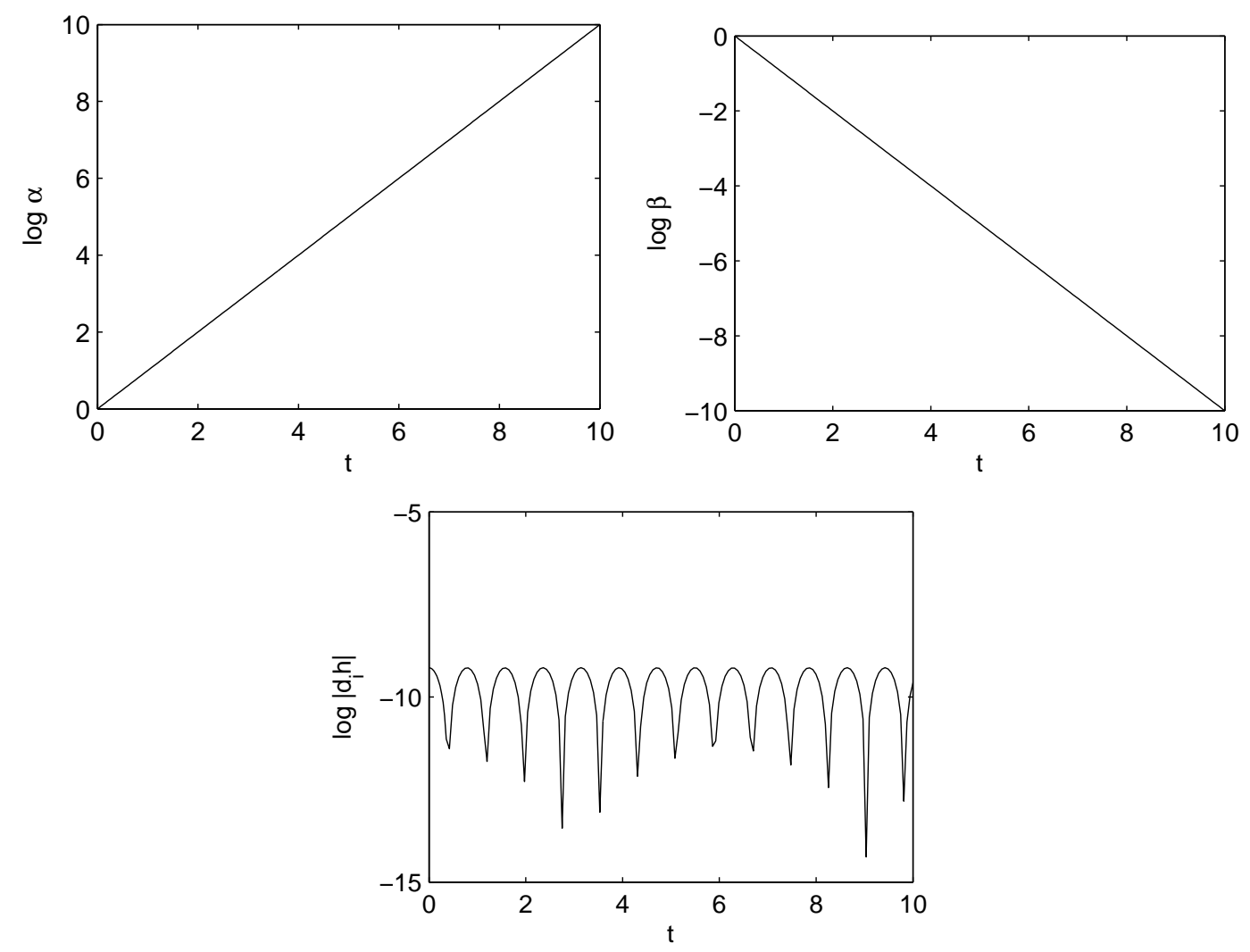

Figure 2: Plots of $\alpha, \beta$ and $h$ for the initial conditions $\alpha_{0}=\beta_{0}=1, \gamma_{0}=0.5, d_{i} f_{0}=-2, d_{i} g_{0}=2$ and $d_{i} h_{0}=10^{-4}$. These initial conditions satisfy the criteria (4.12) and (4.13), hence no finite-time singularity is present and $h(t)$ oscillates about $h=0$.

system but only five equations, so we have to make an assumption for one of the variables in solving the system. The function $\gamma(t)$ is not uniquely determined in our analysis. To find an actual physical form for $\gamma(t)$ we would need another constraint, such as a boundary condition in an initial and boundary value problem or the pressure profile specified by Eq. (3.10). To obtain a numerical solution, we choose $\gamma(t)=$ const for consistency with previous studies [16, 23,37]. Specifically, we choose initial conditions $\alpha_{0}=\beta_{0}=1$ and $\gamma_{0}=0.5$ but vary $f_{0}, g_{0}$ and $h_{0}$. The result (4.29) predicts that $h \rightarrow \infty$ when $\left(h_{0}+\dot{h}_{0} / a\right)>$ 0 , and $h \rightarrow-\infty$ when $\left(h_{0}+\dot{h}_{0} / a\right)<0$. Fig. 2 shows a nonsingular solution, whereas Figs. 3 and 4 show singular solutions when one or both of the conditions (4.12) and (4.13) are not satisfied. The numerical results also show that the accuracy of the predicted value of $t_{s}$ increases as $a^{2}$ increases.

\section{An Alternative Reduction}

In the previous section, we derived an asymptotic solution to the system (3.5)-(3.9), assuming that $\gamma(t)$ remains non-singular. The choice of $\gamma$ was motivated by numerical 

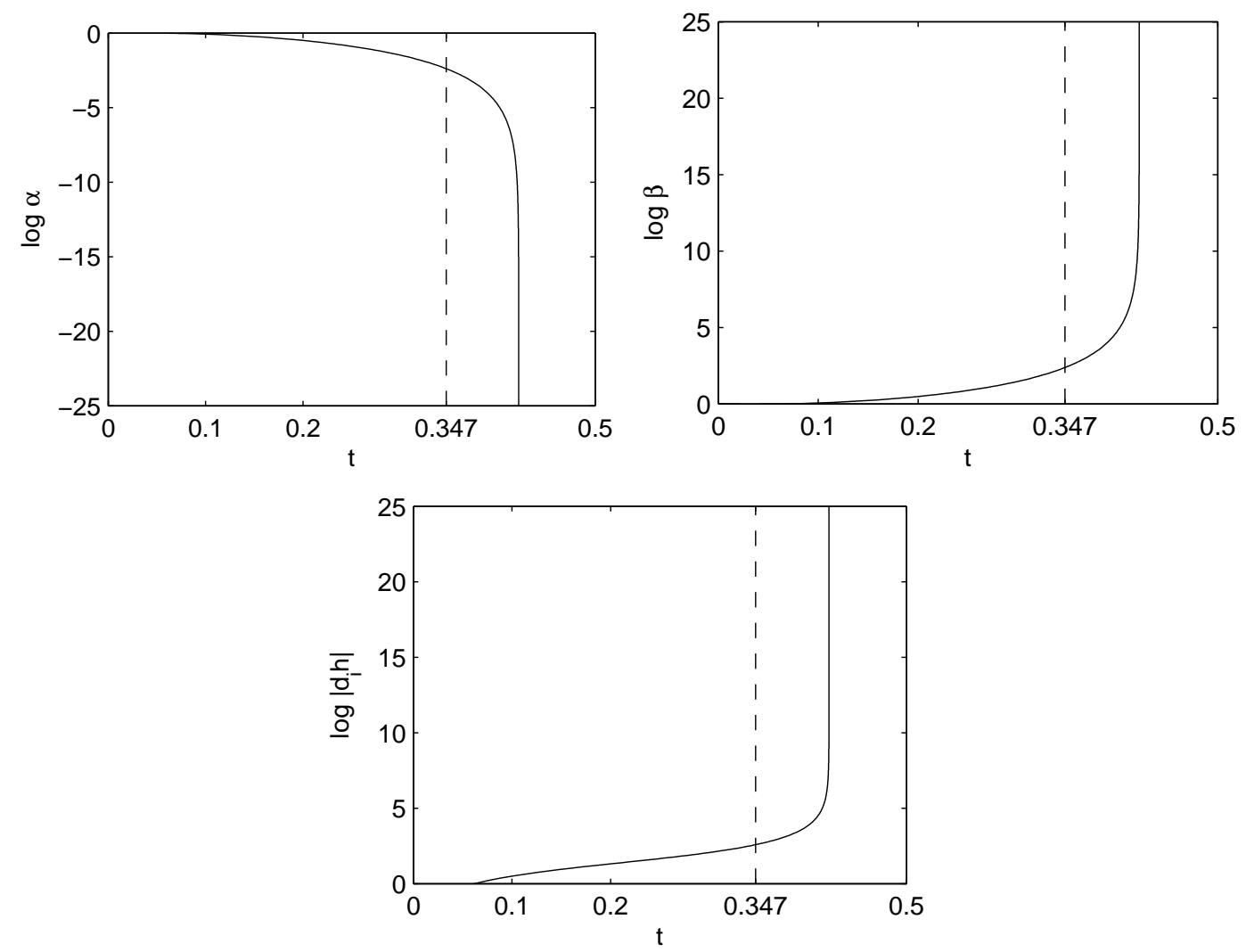

Figure 3: Plots of $\alpha, \beta$ and $h$ for the initial conditions $\alpha_{0}=\beta_{0}=1, \gamma_{0}=0.5, d_{i} f_{0}=2, d_{i} g_{0}=2$ and $d_{i} h_{0}=10^{-4}$ so $a=4$ and $d_{i} \dot{h}_{0}=-16$. Equation (4.29) predicts $h \rightarrow-\infty$ because $h_{0}+\dot{h}_{0} / a<0$. Equation (4.30) predicts the singularity time $t_{s}=0.347$.

MHD simulations [16,37]. For completeness, we now discuss the case of a singular $\gamma(t)$, previously investigated in Ref. [32]. Suppose the pressure is defined by

$$
p(x, y, t)=-\frac{1}{2} h^{2} x^{2} y^{2}+\mu(t)\left(x^{2}+y^{2}\right)
$$

Matching this pressure profile to our general equation for the pressure (3.10) gives

$$
\mu(t)=-4 \alpha(\alpha-\beta)+\gamma^{2}-\dot{\gamma}=4 \beta(\alpha-\beta)-\gamma^{2}-\dot{\gamma},
$$

and rearranging gives an equation for $\gamma(t)$ :

$$
\dot{\gamma}=2\left(\alpha^{2}-\beta^{2}\right) .
$$



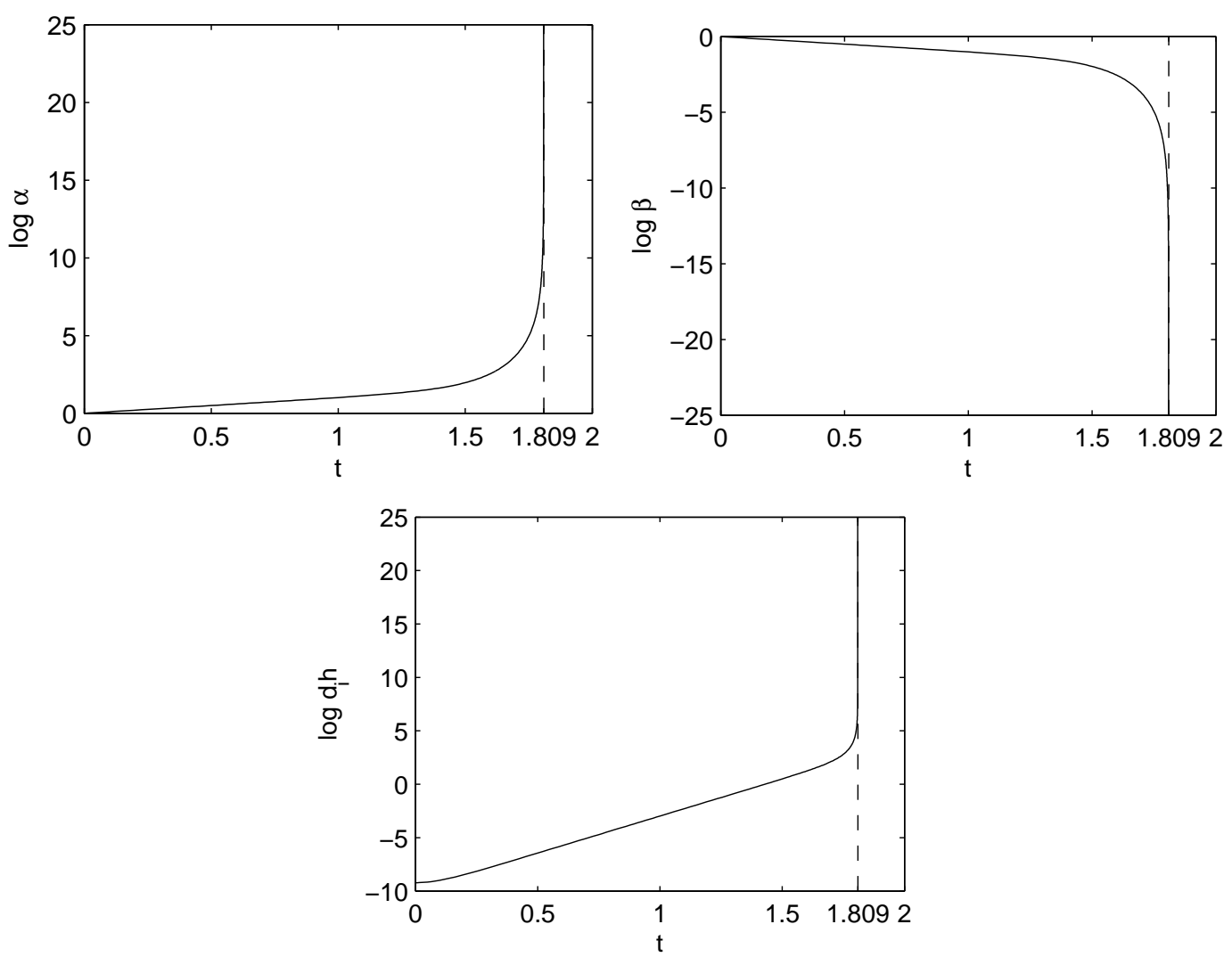

Figure 4: Plots of $\alpha, \beta$ and $h$ for the initial conditions $\alpha_{0}=\beta_{0}=1, \gamma_{0}=0.5, d_{i} f_{0}=2, d_{i} g_{0}=-2$ and $d_{i} h_{0}=10^{-4}$ so $a=4 \sqrt{3}$ and $d_{i} \dot{h}_{0}=0$. Equation (4.29) predicts $h \rightarrow \infty$ because $\left(h_{0}+\dot{h}_{0} / a\right)>0$. Equation (4.30) predicts the singularity time $t_{s}=1.809$.

Suppose also that $\alpha(t)=-d_{i} f(t)$ and $\beta(t)=d_{i} g(t)$. Then the system of equations (3.5)(3.9) becomes

$$
\begin{aligned}
& \dot{\alpha}-2 \alpha\left(\gamma+d_{i} h\right)=0, \\
& \dot{\beta}+2 \beta\left(\gamma+d_{i} h\right)=0, \\
& \dot{\gamma}=2\left(\alpha^{2}-\beta^{2}\right), \\
& d_{i} f=-\alpha, \\
& d_{i} g=\beta, \\
& h=h_{0}=\text { const. . }
\end{aligned}
$$

This system satisfies the conditions (4.12) and (4.13) for $h$ to be non-singular. However, a singularity in $\gamma(t)$ may still lead to a singularity in either $\alpha(t)$ or $\beta(t)$. We can find an equation for $\gamma(t)$ in terms of initial conditions, similar to our treatment of $h(t)$. Differentiating Eq. (5.5) and invoking Eqs. (5.3) and (5.4) yields

$$
\ddot{\gamma}=8\left(\gamma+d_{i} h_{0}\right)\left(\alpha^{2}+\beta^{2}\right),
$$


and differentiating again gives

$$
\dddot{\gamma}=8 \dot{\gamma}\left(\alpha^{2}+\beta^{2}\right)+32\left(\gamma+d_{i} h_{0}\right)^{2}\left(\alpha^{2}-\beta^{2}\right) .
$$

Rearranging Eqs. (5.5) and (5.9) and substituting into Eq. (5.10) yields

$$
\dddot{\gamma}\left(\gamma+d_{i} h_{0}\right)=\dot{\gamma} \ddot{\gamma}+16 \dot{\gamma}\left(\gamma+d_{i} h_{0}\right)^{3},
$$

and on integration

$$
\ddot{\gamma}\left(\gamma+d_{i} h_{0}\right)=\dot{\gamma}^{2}+4\left(\gamma+d_{i} h_{0}\right)^{4}-c,
$$

where $\ddot{\gamma} \gamma$ was integrated by parts. The integration constant is

$$
\begin{aligned}
c= & 4\left(\gamma_{0}+d_{i} h_{0}+\alpha_{0}+\beta_{0}\right)\left(\gamma_{0}+d_{i} h_{0}+\alpha_{0}-\beta_{0}\right) \\
& \times\left(\gamma_{0}+d_{i} h_{0}-\alpha_{0}+\beta_{0}\right)\left(\gamma_{0}+d_{i} h_{0}-\alpha_{0}-\beta_{0}\right) .
\end{aligned}
$$

This reduction was shown to exhibit singularities in purely resistive MHD [30] (when $\left.d_{i} h(t)=0\right)$. Ref. [32] used an exact integral to argue that a large Hall term $\left(d_{i} h_{0} \gg \gamma_{0}\right.$ in our notation) will quench the singularity (see also Refs. [26,31]). However, it is notable that the singularity is still present when the initial values $\gamma_{0}$ and $d_{i} h_{0}$ are comparable, as can be demonstrated by solving Eq. (5.12). Near the singularity, we neglect the integration constant and let $\gamma(t)$ be dependent on a power of $\left(t-t_{0}\right)$ :

$$
\gamma+d_{i} h_{0}=A\left(t-t_{0}\right)^{q}+\ldots
$$

Matching the leading-order terms then yields the solution

$$
\gamma \simeq \pm \frac{1}{2\left(t-t_{0}\right)}-d_{i} h_{0}
$$

which implies a singularity at $t=t_{0}$ unless $\gamma_{0}=-d_{i} h_{0}$. This singularity is illustrated by a numerical solution in Fig. 5.

To sum up, Ref. [32] argues that a large Hall term quenches the singularity in $\gamma(t)$, but we find that the singularity is still present when the values of $d_{i} h_{0}$ and $\gamma_{0}$ are comparable. It may therefore be interesting to investigate the behaviour of the singular solution as $d_{i} h_{0}$ increases.

\section{Resistivity, Viscosity and Electron Inertia}

We generalise the results of the previous sections to include resistivity, viscosity and electron inertia. Assuming $\eta, v$ and $d_{e}$ are not zero in the system (2.1)-(2.6) leads to the system [10]:

$$
\begin{aligned}
& \partial_{t} \psi=-[\psi, \phi]+\eta \nabla^{2} \psi+d_{i}[\psi, Z]+d_{e}^{2}\left(\partial_{t} \nabla^{2} \psi+\left[\nabla^{2} \psi, \phi\right]+[Z, W]\right), \\
& \partial_{t} Z=-[Z, \phi]+\eta \nabla^{2} Z+[W, \psi]+d_{i}\left[\nabla^{2} \psi, \psi\right] \\
& +d_{e}^{2}\left(\partial_{t} \nabla^{2} Z+\left[\nabla^{2} Z, \phi\right]+\left[\nabla^{2} \phi, Z\right]\right), \\
& \partial_{t} W=-[W, \phi]+[Z, \psi]+\nu \nabla^{2} W \text {, } \\
& \partial_{t}\left(\nabla^{2} \phi\right)=-\left[\nabla^{2} \phi, \phi\right]+\left[\nabla^{2} \psi, \psi\right]+\nu \nabla^{4} \phi \text {. }
\end{aligned}
$$




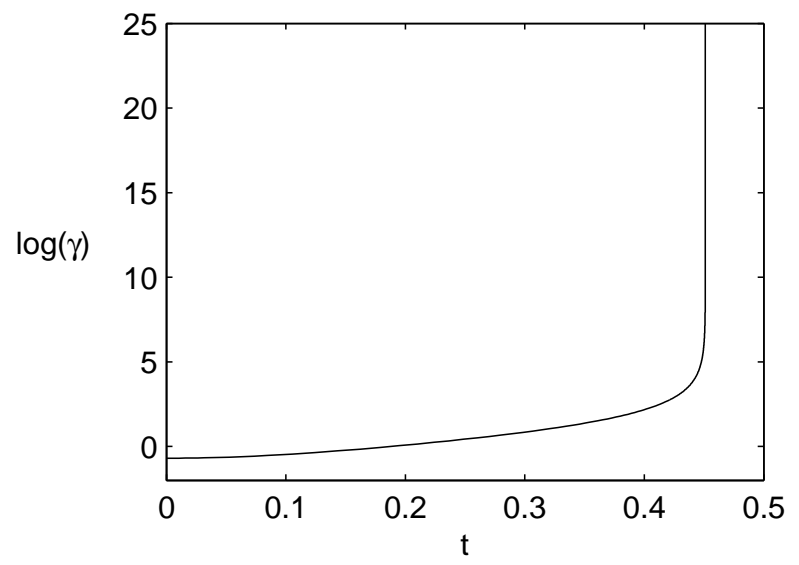

Figure 5: $\gamma(t)$ from the numerical solution of the system of equations (5.3)-(5.5). The initial conditions are $\alpha_{0}=\beta_{0}=1, \gamma_{0}=0.5$ and $d_{i} h_{0}=1$.

Generalising two-dimensional MHD solutions [39], we modify our similarity reduction so that the viscous, resistive and $\partial_{t} \nabla^{2} \psi$ terms cancel when we perform the substitution:

$$
\begin{aligned}
& \psi=\alpha(t) x^{2}-\beta(t) y^{2}+2 \eta \int(\alpha-\beta) \mathrm{d} t+2 d_{e}^{2}(\alpha-\beta), \\
& \phi=-\gamma(t) x y, \\
& W=f(t) x^{2}+g(t) y^{2}+2 v \int(f+g) \mathrm{d} t \\
& Z=h(t) x y .
\end{aligned}
$$

This yields the generalised system

$$
\begin{aligned}
& \dot{\alpha}-2 \alpha\left(\gamma+d_{i} h\right)+2 d_{e}^{2} f h=0, \\
& \dot{\beta}+2 \beta\left(\gamma+d_{i} h\right)+2 d_{e}^{2} g h=0, \\
& \dot{f}-2 \gamma f+2 \alpha h=0, \\
& \dot{g}+2 \gamma g+2 \beta h=0, \\
& \dot{h}+4 \alpha g+4 \beta f=0 .
\end{aligned}
$$

The integrals that generalise Eqs. (4.1) and (4.4) are then

$$
\begin{aligned}
& 4 \alpha \beta=4 \alpha_{0} \beta_{0}+d_{e}^{2}\left(h^{2}-h_{0}^{2}\right), \\
& 4 f g=4 f_{0} g_{0}+\left(h^{2}-h_{0}^{2}\right),
\end{aligned}
$$

and an integral that generalises Eqs. (4.2) and (4.3) is

$$
4\left(\alpha+d_{i} f\right)\left(\beta-d_{i} g\right)=4\left(\alpha_{0}+d_{i} f_{0}\right)\left(\beta_{0}-d_{i} g_{0}\right)+d_{e}^{2}\left(h^{2}-h_{0}^{2}\right) .
$$

To derive a generalised equation for $h$, we differentiate Eq. (6.14) to get

$$
\ddot{h}+8 h\left[d_{i}(\alpha g-\beta f)-2 \alpha \beta-2 d_{e}^{2} f g\right]=0,
$$


and to obtain an expression for $d_{i}(\alpha g-\beta f)$ we use Eq. (6.17). On rearranging terms and using Eqs. (6.15) and (6.16) we get

$$
\ddot{h}-2\left(d_{i}^{2}+4 d_{e}^{2}\right) h^{3}+\left[4 d_{e}^{2}\left(h_{0}^{2}-2 f_{0} g_{0}\right)-a^{2}\right] h=0,
$$

which generalises the Hall MHD result (4.6). The singularity is driven by the nonlinear term, which is proportional to $d_{i}^{2}+4 d_{e}^{2}$. Since $d_{e}^{2} / d_{i}^{2}=m_{e} / m_{i} \ll 1$, we conclude that electron inertia is unlikely to modify the X-point collapse in a significant manner. However, once a large electric current density is reached at the magnetic null and the self-similar Hall MHD solution breaks down, higher-order terms in the induction equation (say due to hyper-resistivity [45] or off-diagonal terms in the electron pressure tensor [6]) will control the structure of the current sheet on the electron scale $\sim d_{e}$. Description of that structure is beyond the scope of our self-similar collapse model.

\section{Discussion}

The predicted collapse time $t_{s}$ decreases if the strength of the Hall term quantified by the ion skin depth $d_{i}$ increases. This result is consistent with numerical solutions $[2,3,12$, $22,29]$, which show that the Hall effect speeds up the reconnection process. In the limit $d_{i} \rightarrow 0$, the singularity formation time $t_{s} \rightarrow \infty$ corresponds to the well-established absence of a finite-time singularity in ideal MHD collapse.

Due to the geometry we have used, our solution has no spatial dependence on resistivity. A more general geometry could mean that the singularity is arrested by the resistivity. Steady resistive Hall MHD solutions $[10,11]$ based on a one-dimensional planar magnetic field (as opposed to the X-point geometry of our solution) do possess a resistive scale. In the context of a general initial and boundary value problem, our solution can be considered to be a low-order Taylor expansion of the flux and stream functions at the origin. This approximation implies that the solution only holds locally and breaks down before the singularity is reached, so we can no longer describe the current sheet structure. The structure will be described by a steady model that has a small resistive scale, as argued for instance in Ref. [15]. As in the corresponding MHD solutions [8, 40], another limitation for the solution to be valid in a resistive plasma is that a specific varying electric field must be applied, proportional to the plasma resistivity. Furthermore, as pointed out by a referee, the singularity could be arrested by an acceleration such as gravity, although the corresponding Rayleigh-Taylor Hall instability can be quite fast $[18,42]$. However, despite the limitations of the model the value of our detailed calculation is that the formula for the singularity formation time quantifies the role of the Hall effect and initial conditions in the current sheet formation.

Our solution may be applicable in a weakly collisional plasma of the solar corona, where the reference values of $L=10^{9.5} \mathrm{~cm}, B_{0}=10^{2} \mathrm{G}$ and $n=10^{9} \mathrm{~cm}^{-3}$ yield the dimensionless ion skin depth $d_{i} \sim 10^{-6.5}$. If the Sweet-Parker length scale $\eta^{1 / 2}$ is based on the collisional resistivity $\eta \sim T^{-3 / 2}$ [36], then the coronal temperature $T=10^{6} \mathrm{~K}$ gives $\eta \sim 10^{-14.5}$ and so the Hall term dominates $\left(d_{i} \gg \eta^{1 / 2}\right)$ [9]. Cassak et al. [5] argue that an explosive character 
of magnetic reconnection in solar flares can be explained by a rapid transition from slow Sweet-Parker reconnection to fast Hall reconnection in an evolving current sheet. The solution presented here models such a rapid transition as a singularity formation at time $t_{s}$. Assuming $a \sim h_{0} \sim 1$, our solution predicts the transition time $t_{s} \sim 10 t_{A}$, where the Alfvén time $t_{A}=L / v_{A}=10^{0.5}$ seconds. This estimate is consistent with typical flare onset times and simulation results [5].

To conclude, we have presented a self-similar solution for current sheet formation at a magnetic neutral line in incompressible Hall MHD, generalising previous studies [23] by considering a general set of initial conditions. A criterion for finite-time singularity formation that describes the collapse to a current sheet was derived, and we illustrated both the criterion and predicted collapse time with numerical solutions of the Hall MHD equations. Finally, we generalised the self-similar solution to incorporate electron inertia, resistive and viscous terms in Ohm's law and the equation of motion.

\section{Acknowledgments}

We thank Professors Ian Craig and Roger Hosking, and also the anonymous referees, for useful comments and suggestions.

\section{References}

[1] A. Bhattacharjee, Impulsive magnetic reconnection in the Earth's magnetotail and the solar corona, Annu. Rev. Astron. Astrophys. 42, 365-384 (2004).

[2] J. Birn, J.F. Drake, M.A. Shay, B.N. Rogers, R.E. Denton, M. Hesse, M. Kuznetsova, Z.W. Ma, A. Bhattacharjee, A. Otto and P.L. Pritchett, Geospace Environmental Modeling (GEM) magnetic reconnection challenge, J. Geophys. Res. 106, 3715-3720 (2001).

[3] J. Birn, K. Galsgaard, M. Hesse, M. Hoshino, J. Huba, G. Lapenta, P.L. Pritchett, K. Schindler, L. Yin, J. Büchner, T. Neukirch and E.R. Priest, Forced magnetic reconnection, Geophys. Res. Lett. 32, 6105 (2005).

[4] S.V. Bulanov and M.A. Olshanetskii, Magnetic collapse near zero points of the magnetic field, Phys. Letts. 100A, 35-38 (1984).

[5] P.A. Cassak, J.F. Drake and M.A. Shay, A model for spontaneous onset of fast magnetic reconnection, Astrophys. J. 644, L145 (2006).

[6] P. A. Cassak, R. N. Baylor, R. L. Fermo, M. T. Beidler, M. A. Shay, M. Swisdak, J. F. Drake and H. Karimabadi, Fast magnetic reconnection due to anisotropic electron pressure, Phys. Plasmas 22, 020705 (2015).

[7] S. Chapman and P.C. Kendall, Liquid instability and energy transformation near a magnetic neutral line: A soluble non-linear hydromagnetic problem, Proc. Roy. Soc. London, Ser. A 271, 435-448 (1963).

[8] S. Chapman and P.C. Kendall, Comment on "Some Exact Solutions of Magnetohydrodynamics", Phys. Fluids 9, 2306-2307 (1966).

[9] I.J.D. Craig and Y.E. Litvinenko, Influence of the Hall effect on the reconnection rate at line-tied magnetic X-points, Astron. Astrophys. 484, 847-850 (2008).

[10] I.J.D. Craig and P.G. Watson, Exact models for Hall current reconnection with axial guide fields, Phys. Plasmas 12, 012306 (2005). 
[11] J.C. Dorelli, Effects of Hall electric fields on the saturation of forced antiparallel magnetic field merging, Phys. Plasmas 10, 3309-3314 (2003).

[12] J.F. Drake, M.A. Shay and M. Swisdak, The Hall fields and fast magnetic reconnection, Phys. Plasmas 15, 042306 (2008).

[13] J. Dungey, Conditions for the occurrence of electrical discharges in astrophysical systems, Phil. Mag. 44, 725-738 (1953).

[14] J.W. Dungey, The neutral point discharge theory of solar flares. A reply to Cowling's criticism, in Electromagnetic Phenomena in Cosmical Physics, IAU Symp., vol. 6, edited by B. Lehnert, pp. 135-140 (1958).

[15] T.G. Forbes, Implosion of a uniform current sheet in a low-beta plasma, J. Plasma Phys. 27, 491-505 (1982).

[16] R. Grauer and C. Marliani, Geometry of singular structures in magnetohydrodynamic flows, Phys. Plasmas 5, 2544 (1998).

[17] E.G. Harris, On a plasma sheath separating regions of oppositely directed magnetic field, Nuovo Cimento 23, 115-121 (1962).

[18] R.J. Hosking, New instabilities due to Hall effect, Phys. Rev. Lett. 15, 344-345 (1965).

[19] R.J. Hosking and G.M. Marinoff, Magneto-viscous effects on the ideal and resistive gravitational instabilities in Cartesian geometry, Plasma Phys. 15, 327-341 (1973).

[20] V.S. Imshennik and S.I. Syrovatskii, Two-dimensional flow of an ideally conducting gas in the vicinity of the zero line of a magnetic field, Sov. Phys.-JETP 25, 656 (1967).

[21] I. Klapper, Constraints on finite-time current sheet formation at null points in two-dimensional ideal incompressible magnetohydrodynamics, Phys. Plasmas 5, 910-914 (1998).

[22] D.A. Knoll and L. Chacón, Coalescence of magnetic islands in the low-resistivity, Hall-MHD regime, Phys. Rev. Lett. 96, 135001 (2006).

[23] Y.E. Litvinenko, Current sheet formation at a magnetic neutral line in Hall magnetohydrodynamics, Phys. Plasmas 14, 112303 (2007).

[24] L.M. Malyshkin, Model of Hall reconnection, Phys. Rev. Lett. 101, 225001 (2008).

[25] J.A. McLaughlin, A.W. Hood and I. de Moortel, MHD wave propagation near coronal null points of magnetic fields, Space Sci. Rev. 158, 205-236 (2011).

[26] M. Núñez, J. Álvarez and J. Rojo, Blowup of certain analytic solutions of the Hall magnetohydrodynamic equations, Phys. Plasmas 15, 062104 (2008).

[27] E.N. Parker, Sweet's mechanism for merging magnetic fields in conducting fluids, J. Geophys. Res. 62, 509-520 (1957).

[28] B. Rossi and S. Olbert, Introduction to the Physics of Space, McGraw-Hill, New York (1970).

[29] M.A. Shay, J.F. Drake, B.N. Rogers and R.E. Denton, Alfvénic collisionless magnetic reconnection and the Hall term, J. Geophys. Res. 106, 3759-3772 (2001).

[30] B.K. Shivamoggi, Evolution of current sheets near a hyperbolic magnetic neutral point, Phys. Fluids 29, 769-772 (1986).

[31] B.K. Shivamoggi, Hall magnetohydrodynamics near an X-type magnetic neutral line, Europhys. Lett. 85, 25001 (2009).

[32] B.K. Shivamoggi, Steady and unsteady Hall magnetohydrodynamics near an X-type magnetic neutral line, Phys. Plasmas 18, 052304 (2011).

[33] A.N. Simakov and L. Chacón, Quantitative analytical model for magnetic reconnection in Hall magnetohydrodynamics, Phys. Plasmas 16, 055701 (2009).

[34] R. Smets, N. Aunai, G. Belmont, C. Boniface and J. Fuchs, On the relationship between quadrupolar magnetic field and collisionless reconnection, Phys. Plasmas 21, 062111 (2014).

[35] B.U.Ö. Sonnerup, Magnetic field reconnection, in Solar System Plasma Physics, vol. 3, L.J. Lanzerotti, C.F. Kennel and E.N. Parker (eds.), pp. 45-108, North-Holland, Amsterdam 
(1979).

[36] L. Spitzer, Physics of Fully Ionized Gases, 2 ed., Interscience, New York (1962).

[37] P.L. Sulem, U. Frisch, A. Pouquet and M. Meneguzzi, On the exponential flattening of current sheets near neutral X-points in two-dimensional ideal MHD flow, J. Plasma Phys. 33, 191-198 (1985).

[38] P.A. Sweet, The neutral point theory of solar flares, in Electromagnetic Phenomena in Cosmical Physics, IAU Symp., vol. 6, edited by B. Lehnert, pp. 123-134, Cambridge University Press, London (1958).

[39] M.S. Uberoi, Some exact solutions of magnetohydrodynamics, Phys. Fluids 6, 1379-1381 (1963).

[40] M.S. Uberoi, Reply to comments by S. Chapman and P. C. Kendall, Phys. Fluids 9, 2307 (1966).

[41] D.A. Uzdensky, On the physical interpretation of Malyshkin's (2008) model of resistive Hall magnetohydrodynamic reconnection, Phys. Plasmas 16, 040702 (2009).

[42] A.L. Velikovich, Rayleigh-Taylor instability of a plasma-vacuum boundary in the limit of a large Larmor radius, Phys. Fluids B 3, 492-494 (1991).

[43] X. Wang, A. Bhattacharjee and Z.W. Ma, Collisionless reconnection: Effects of Hall current and electron pressure gradient, J. Geophys. Res. 105, 27633-27648 (2000).

[44] M. Yamada, R. Kulsrud, and H. Ji, Magnetic reconnection, Rev. Mod. Phys. 82, 603 (2010).

[45] L. Zocco, A. Chacón and A.N. Simakov, Current sheet bifurcation and collapse in electron magnetohydrodynamics, Phys. Plasmas 16, 110703 (2009).

[46] E.G. Zweibel and M. Yamada, Magnetic reconnection in astrophysical and laboratory plasmas, Annu. Rev. Astron. Astrophys. 47, 291-332 (2009). 